\title{
Diffractive PDFs and factorisation tests at HERA
}

\author{
Alberto GARFAGNINI ${ }^{* \dagger}$ \\ Università di Padova and INFN, sezione di Padova \\ E-mail: alberto.garfagnini@pd.infn.it
}

The HERA collider at DESY (Hamburg) has provided Deep Inelastic Scattering (DIS) of electrons off protons in the years 1992-2007 for the H1 and ZEUS experiments with a centre of mass energy up to $318 \mathrm{GeV}$. Diffractive dissociation of photons in the reaction $\gamma^{(*)} p \rightarrow X p$, constitute a large fraction $(\sim 10 \%)$ of the visible cross section in deep inelastic scattering (DIS), and the process can be seen as a reaction where the colliding proton emerges intact in the interaction. Different experimental methods have been developed by the $\mathrm{H} 1$ and ZEUS experiments to select diffractive events: by detecting the final state proton with forward proton spectrometers or thanks to to the peculiar topology of diffractive events, by requiring a large rapidity gap in the main detector between the leading proton (or the low-mass baryonic system) and the photon dissociation system X. The ZEUS and H1 collaborations have performed next-to-leading-order (NLO) QCD fit to the inclusive diffractive data extracting diffractive parton densities (DPDFs). As a direct test of the QCD factorisation theorem, dijet cross sections have been compared to the NLO order QCD predictions, based on recent DPDFs obtained by $\mathrm{H} 1$ and ZEUS. New sets of DPDFs are obtained through a simultaneous fit to the inclusive and dijet diffractive cross sections. The analysis allows for a precise determination of both the quark and gluon distributions over a wide $z$ range: the precision on the gluon density at high momentum fractions is improved compared to previous extractions since data on diffractive dijets allow to constrain the gluon density. Finally, recent factorization tests on diffractive dijets in photoproduction are presented and discussed in the following.

European Physical Society Europhysics Conference on High Energy Physics July 16-22, 2009

Krakow, Poland

* Speaker.

$\dagger$ On behalf or the H1 and ZEUS Collaborations 


\section{Diffractive Parton Densities from Inclusive Data}

The hard scattering QCD factorisation theorem [1] for semi-inclusive DDIS processes allows to introduce the concept of diffractive parton distribution functions (DPDFs): the latter represent conditional parton probability distributions under the constraint of a leading proton in the final state with a well defined four-momentum. The differential DDIS cross section may be written as follows

$$
d \sigma^{e p \rightarrow e p X}\left(x, Q^{2} ; x_{I P}, t\right)=\sum_{i} f_{i}^{D}\left(x, Q^{2} ; x_{I P}, t\right) \times d \hat{\sigma}^{e i}\left(x, Q^{2}\right),
$$

where $\hat{\sigma}^{e i}\left(x, Q^{2}\right)$ is the hard-scattering partonic cross section, while $f_{i}^{D}$ is the DPDFs for the parton $i$. (Details on the Deep Inelastic Scattering variables used at HERA can be found in [2] or [3].) A "Proton Vertex Factorization" hypothesis has been verified by both H1 [2] and ZEUS [3] diffractive data: the observables related to the scattered proton $\left(x_{I P}, t\right)$ factorise from those describing the hard partonic interaction $\left(Q^{2}, \beta\right)$. Therefore, it is possible to to rewrite the DPDFs as

$$
f_{i}^{D}\left(x, Q^{2} ; x_{I P}, t\right)=f_{I P / p}\left(x_{I P}, t\right) \cdot f_{i}\left(\beta=x / x_{I P}, Q^{2}\right) .
$$

The ZEUS collaboration has recently performed a Next-to-Leading-Order QCD fits to the inclusive DDIS data selected with the LRG and LPS methods [4]. For the first time ever the heavy quark contribution was treated within the general-mass variable flavour-number scheme of Thorne and Roberts (TR-VFNS) [5], while the influence of $F_{L}^{D(3)}$ was accounted for through its NLO dependence on the parton densities. The analysis made use of the proton vertex factorization hypothesis, with the $x_{I P}$ dependence of the DPDFs parametrized with Regge inspired Pomeron and Reggeon fluxes (with linear trajectories). While the quark densities are well described by the data and little discrepancies are seen between the different fits, the gluon density has large uncertainties, especially at high $z$, since the gluon is only indirectly constrained by the scaling violations $\partial F_{2}^{D} / \partial \ln Q^{2}$. Similar fits have been performed by H1 on DDIS [2] achieving similar results.

\section{Diffractive Parton Densities and Diffractive Dijets}

According to the QCD factorization theorem [1], the diffractive parton densities extracted from the inclusive data should be applicable to the prediction of other observables. As a direct application, factorization tests can be performed on dijet production and heavy flavour in diffractive scattering. Since the main mechanism for dijet production at HERA, is the boson-gluon fusion process where at leading order gluons from the proton directly interact with the photon creating dijets, dijet data are directly sensitive to the diffractive gluon density. The distributions have been compared to the NLO QCD predictions using the H1 [2] and ZEUS [4] DPDFs with similar results and indicating that the data show a high sensitivity to the gluon density. New QCD fits have been performed by both H1 [6] and ZEUS [7] to the DDIS simultaneously to the inclusive and dijet data: the calculations allow to constrain both the quark and gluon distributions with comparable precisions across the whole kinematic range. Recent factorization tests have been performed by ZEUS applying the DPDFs to NLO predictions for the charm diffractive structure function [8]. As can be seen from Fig. 1, the calculations give a a fair agreement with the data (which are still statistically limited). 



Figure 1: ZEUS Diffractive Charm data compared to NLO calculations with DPDFs from djet+inclusive data.

\section{Diffractive Dijet in Photoproduction}

The NLO calculations with DPDFs from the inclusive and dijet data have been further compared to hard diffractive photoproduction reactions. In the latter case the photon can develop an effective "partonic structure" fluctuating in $q \bar{q}$ pairs. In the simple leading order picture, the photon can "resolve" and only a fraction $x_{\gamma}$ of its four-momentum participate in the hard subprocess $\left(x_{\gamma}<1\right)$, or can interact as a point-like "direct" particle $\left(x_{\gamma}=1\right)$. The question on the universalities of the DPDFs has been address by the H1 and ZEUS collaborations. Fig. 2 shows the H1 [9] (left) and ZEUS [10] (right) measurements of diffractive dijet cross section in photoproduction as a function of $x_{\gamma}$. The NLO calculationsuse the H1 2006 Fit B DPDFs for the H1 data (left plot) and the new H1 Fit 2007 and ZEUS DPDF SJ for the ZEUS data (right plot). Both measurements have been performed in similar kinematic regions and even if a small suppression of the resolved component is seen for the $\mathrm{H} 1$ data, the data are compatible. The ratios of data to theory measured by both experiments show a very weak dependence on $x_{\gamma}$ which is in contrast to some theoretical expectations [11].But since the correlations between the involved observables are complicated - for instance $E_{T}^{j e t 1}$ and $x_{\gamma}$ are strongly correlated - more differential studies are required to fully unfold the underlying dynamics.

\section{Conclusions}

A wealth of data has been published in recent years on diffraction by the H1 and ZEUS collaborations, using different techniques for the selection of diffractive events. The Proton Vertex Factorization hypothesis, well supported by the measured observable, allows to extract diffractive parton densities from NLO QCD fits to the inclusive data. Diffractive dijet data are a suitable test bench for the universalities of the parton densities and due to their main production mechanism (boson-gluon fusion), are very sensitive to the diffractive gluon densities. Combined fits to inclusive and dijet data allow to constrain simultaneously the quark and gluon densities to good and similar precisions. Finally the DPDF predictions compared to the hard diffractive photoproduc- 



Figure 2: Top: measurement of the differential cross section $d \sigma / d x_{\gamma}$ for diffractive dijets in photoproduction for H1 (left) and ZEUS (right). Bottom: ratio of the measured cross section to the NLO calculations. For the H1 data (left) the H1 2006 Fit B DPDFs are used. For the ZEUS data (right) the new H1 Fit 2007 and ZEUS DPDF SJ DPDFs have been exploited.

tion data are very similar for direct and resolved photon interactions, a fact which deserves further experimental and theoretical investigations.

\section{References}

[1] J. C. Collins, Phys. Rev. D 57, 3051 (1998); Erratum, Phys. Rev. D 61, 019902 (2000).

[2] H1 Coll., A. Aktas et al., Eur. Phys. J. C 48, 715 (2006); H1 Coll., Eur. Phys. J. C 48, 749 (2006).

[3] ZEUS Coll., S. Chekanov et al., Nucl. Phys. B 816, 1 (2009)

[4] ZEUS Coll, "A QCD analysis of diffractive deep inelastic scattering at HERA", ZEUS-prel-09-004.

[5] R, S. Thorne and R. G. Roberts Phys. Rev. D 57, 6871 (1998).

[6] H1 Coll., Eur. Phys. J. C 20, 29 (2001).

H1 Coll., A. Aktas et al., JHEP 0710, 042 (2007).

[7] ZEUS Coll., S. Chekanov et al., Eur. Phys. J. C 52, 813 (2007).

[8] ZEUS Coll., S. Chekanov et al., Nucl. Phys. B 672, 3 (2003).

[9] H1 Coll., "Diffractive photoproduction of jest with the H1 detector", H1prelim-08-012.

[10] ZEUS Coll., S. Chekanov et al., Eur. Phys. J. C 55, 177 (2008).

[11] A. Kaidalov, V. Khoze, A. Martin, M. Ryskin, Phys. Lett. B 567, 61 (2003). M. Klasen, G. Kramer, Mod. Phys. Lett. A 23, 1885 (2008). 